Editor's Note: These short reviews of a recent paper in the Journal, written exclusively by graduate students or postdoctoral fellows, are intended to mimic the journal clubs that exist in your own departments or institutions. For more information on the format and purpose of the Journal Club, please see http://www.jneurosci.org/misc/ifa_features.shtml.

\title{
What You Set Is Not What You See: Unconscious Activation of Cognitive Control
}

\author{
Carly J. Leonard and Yu-Chin Chiu \\ Department of Psychological and Brain Sciences, The Johns Hopkins University, Baltimore, Maryland 21218 \\ Review of Lau and Passingham (http://www.jneurosci.org/cgi/content/full/27/21/5805)
}

Cognitive control can prevent purely stimulus-driven or reflexive processing from dominating neural representation, thought, and behavior. This top-down cognitive control instead allows for goaldriven attentional selection and taskrelevant behavior. Consciousness is often assumed to be intrinsic to these high-level control functions sitting atop a processing hierarchy in the prefrontal cortex (PFC). However, in a recent The Journal of Neuroscience article, Lau and Passingham (2007) present new evidence that questions this assumption.

Preparation for an upcoming task is an important element of cognitive control. Before execution, neural activity increases in cortical regions that process information relevant to the upcoming task. For example, cues indicating an upcoming color task initiate activation of visual areas that process color information (Wylie et al., 2006). This finding suggests that taskset preparation may use mechanisms similar to those in the biased-competition model of attention (Desimone and Duncan, 1995), in which selective attention modulates lower visual area processing via top-down biasing. Extending the concept of biased competition outside the visual domain, cortical regions associated with

Received July 26, 2007; revised Aug. 29, 2007; accepted Sept. 3, 2007. Correspondence should be addressed to Carly J. Leonard, Department of Psychological and Brain Sciences, The Johns Hopkins University, Ames Hall, 3400 North Charles Street, Baltimore, MD 21218. E-mail: carly@jhu.edu. D01:10.1523/JNEUROSCI.3394-07.2007

Copyright $\odot 2007$ Society for Neuroscience $\quad 0270-6474 / 07 / 2711170-02 \$ 15.00 / 0$ task processing may also receive topdown modulation. The PFC is considered as the source of both attentional and taskset control signals.

Preparing for a specific task can result in task-specific processing of even unseen stimuli. Naccache et al. (2002) engaged participants in a number judgment task. A masked number prime was presented before the target number for which the task was intended. Even when the masked number was unable to be seen, priming was observed, showing that semantic analysis and response generation occur even for a stimulus that does not enter conscious awareness. Lau and Passingham (2007) take this one step further and illustrate how unseen cues can interfere with task-set control.

The design of Lau and Passingham (2007) takes advantage of metacontrast masking, as well as two tasks that involve discrete brain regions, specifically left inferior frontal cortex and middle temporal gyrus for semantic judgments and left ventral premotor area for phonological judgments (Sakai and Passingham, 2006). Metacontrast masking occurs when an object is rendered invisible by a subsequently presented mask containing adjacent contours. The metacontrast masking used by Lau and Passingham (2007) had an interesting temporal profile, such that the prime was less visible when the stimulus onset asynchrony (SOA) between the prime and the cue (which also served as a mask) was longer. Two SOAs between the prime and cue were chosen: $83 \mathrm{~ms}$ (LowVisibility condition), in which the cue acted as an effective metacontrast mask of the prime, and $16 \mathrm{~ms}$ (High-Visibility condition), in which it did not. On each trial, participants were cued by a diamond or square as to which task to perform on an upcoming word. The primes were smaller versions of the cues and thus also had associations with task set [Lau and Passingham (2007), their Fig. 1 (http:// www.jneurosci.org/cgi/content/full/27/ $21 / 5805 / F 1)]$. Congruency between the prime and the cue was manipulated, such that on some trials they were associated with the same task set, whereas on others they were not. Functional magnetic resonance imaging data were collected from 12 participants.

To ensure the validity of the visibility manipulation, the discriminability of the two primes at each SOA was examined for each subject. Data from two subjects were discarded because of better performance in the Low-Visibility condition, inconsistent with the intent of the manipulation. For the remaining subjects, discriminability was not significantly different from zero in the Low-Visibility condition.

Both behavioral and imaging data from the main experiment suggest that subliminal priming occurred at the taskset level. Incongruent primes impaired performance on both accuracy and reaction time measures [Lau and Passingham (2007), their Fig. 2 (http://www. jneurosci.org/cgi/content/full/27/21/ 
5805/F2)]. This effect was only observed in the Low-Visibility condition, indicating that it was subliminal. Furthermore, this subliminal priming was associated with an increase in neural activity in the task-irrelevant cortical region and a decrease in the task-relevant cortical region [Lau and Passingham (2007), their Fig. 3 (http://www.jneurosci.org/cgi/content/ full/27/21/5805/F3)]. Right dorsolateral PFC (DLPFC) also showed this subliminal priming [Lau and Passingham, their Fig. 4 (http://www.jneurosci.org/cgi/content/ full/27/21/5805/F4)]. This may have reflected the detection of conflict between the prime and cue or the increased demand for two different task sets.

Because the current study only showed a priming effect in one direction (i.e., interference), future work could add a neutral prime that does not map onto any task set. This manipulation would provide a baseline for the congruent and incongruent conditions that distinguishes the two possible roles for DLPFC. If the increased DLPFC activity is caused by conflict, differential activity there would not be expected in a congruent/neutral contrast. Also, if priming is possible at the task-set control level, one might expect to see facilitation from congruent primes, not just interference from incongruent primes.

The idea of unconscious priming at the task-set level challenges the conventional view that cognitive control requires consciousness and attention. Here, Lau and Passingham (2007) suggest that consciousness is not critical to cognitive control. However, attention may well be. Naccache et al. (2002) suggested that attention during the time interval containing the prime is critical for eliciting priming. Likewise, throughout the current experiment, participants were required to discriminate the briefly presented cues and thus had an attentional set for these cues. In this way, unconscious primes may only gain access to task-set control mechanisms because of top-down biasing for this particular attentional set. Nonetheless, one might wonder whether subliminal task-set interference would remain as strong if task set had been specified before the presentation of an unconscious prime. Preparing an attentional set can prevent distractor interference (for review, see Egeth and Yantis, 1997), which may hold true for task set as well, if the biasedcompetition model explains both attention and task set.

On the one hand, overlearned associations can be enacted quickly and reliably without the need for extended deliberation. On the other hand, unexpected changes in the environment may demand time-consuming but flexible cognitive control. The human brain must have evolved to strike a balance between flexibility and stability, allowing for a dynamic equilibrium that does not rely on one discrete mode or the other. The results of Lau and Passingham (2007) established an important role for unconscious priming in this dynamic system of cognitive control. Ultimately, top-down and bottom-up control of attention and task set determines which stimuli gain access to further processing and what kind of processing will be applied to them.

\section{References}

Desimone R, Duncan J (1995) Neural mechanisms of selective visual attention. Annu Rev Neurosci 18:193-222.

Egeth HE, Yantis S (1997) Visual attention: control, representation, and time course. Annu Rev Psychol 48:269-297.

Lau HC, Passingham RE (2007) Unconscious activation of the cognitive control system in the human prefrontal cortex. J Neurosci 27:5805-5811.

Naccache L, Blandin E, Dehaene S (2002) Unconscious masked priming depends on temporal attention. Psychol Sci 13:416-424.

Sakai K, Passingham RE (2006) Prefrontal set activity predicts rule-specific processing during subsequent cognitive performance. J Neurosci 26:1211-1218.

Wylie GR, Javitt DC, Foxe JJ (2006) Jumping the gun: is effective preparation contingent upon anticipatory activation in task-relevant neural circuitry? Cereb Cortex 16:394-404. 